\title{
Camilo José Vergara, un photographe « scientifique » et politique
}

Camilo José Vergara, a «scientific " and political photographer

Camilo José Vergara, un fotógrafo « científico » y político

\section{Philippe Bazin}

\section{OpenEdition}

\section{Journals}

Édition électronique

URL : http://journals.openedition.org/ideas/5135

DOI : 10.4000/ideas. 5135

ISSN : 1950-5701

\section{Éditeur}

Institut des Amériques

\section{Référence électronique}

Philippe Bazin, «Camilo José Vergara, un photographe « scientifique » et politique », IdeAs [En ligne] 13 | 2019, mis en ligne le 01 mars 2019, consulté le 19 avril 2019. URL : http:// journals.openedition.org/ideas/5135; DOI : 10.4000/ideas.5135

Ce document a été généré automatiquement le 19 avril 2019.

\section{cc) (†) $\odot$}

IdeAs - Idées d'Amériques est mis à disposition selon les termes de la licence Creative Commons Attribution - Pas d'Utilisation Commerciale - Pas de Modification 4.0 International. 


\title{
Camilo José Vergara, un photographe «scientifique » et politique
}

\author{
Camilo José Vergara, a «scientific " and political photographer \\ Camilo José Vergara, un fotógrafo « científico " y político
}

\section{Philippe Bazin}

\begin{abstract}
"Il est plus difficile d'honorer la mémoire des sans-nom que celle des gens reconnus. À la mémoire des sans-nom est dédiée la construction historique." Walter Benjamin (Benjamin W., 1991 : 356) «Il n'y a pas lieu de craindre ou d'espérer, mais de chercher de nouvelles armes. " Gilles Deleuze, « Post-scriptum sur les sociétés de contrôle » (in Antonioli M., 2015 : 13)
\end{abstract}

\section{Introduction}

1 Camilo José Vergara, photographe et sociologue chilien émigré aux États-Unis, a accompli depuis le milieu des années 1970 un ample et unique travail de recherche et de création dont le support principal croise la photographie et le texte. Sa démarche peut être qualifiée de «scientifique». Plusieurs publications en attestent qui montrent le lieu presque exclusif de son travail, les ghettos noirs américains. Pendant plus de quarante ans, il a choisi un certain nombre de lieux spécifiques sur lesquels il revient régulièrement photographier. Cette régularité lui a permis d'utiliser une triple méthodologie de travail.

2 Tout d'abord, il use, comme nul autre dans la photographie de son époque, de la reconduction photographique qui permet sur un même site la visualisation au long cours de l'évolution du ghetto. 
3 De plus, cela lui permet de comparer les évolutions des différents ghettos auxquels il s'est intéressé par le biais d'une transversalité photographique qui met en confrontation ceuxci à la même époque, repérant par là des phénomènes similaires à des milliers de kilomètres de distance. Ces usages de la transversalité et de la reconduction lui permettent de mettre en évidence à la fois des politiques ponctuelles et de longue durée menées par les gouvernements américains, ainsi que la manière dont les habitants s'organisent pour y répondre.

4 Enfin, Vergara définit très clairement les différents éléments de sa méthodologie dans son ouvrage séminal, The New American Ghetto (1995), donnant une liste précise des points sur lesquels s'appuie conceptuellement son travail, ces points entremêlant pratiques photographique et sociologique.

5 Pour donner une vision d'ensemble de son projet artistique et sociologique, Vergara utilise à partir des années 2000 les moyens informatiques de classement, pour mettre en évidence ce qui constitue la forme esthétique de son œuvre, l'archive. Ainsi ne constitue$\mathrm{t}$-il pas seulement une collection, mais une archive interactive sans cesse en mouvement avec le temps. Plusieurs sites web en témoignent qui doivent être compris comme de véritables outils de recherche.

Depuis 1977, Vergara documente la destruction urbaine à travers tous les États-Unis, constituant une archive qui inclut le South Bronx, Harlem, et North Central Brooklyn ; Newark et Camden dans le New Jersey ; Chicago ; Gary dans l'Indiana ; Détroit ; et le comté de Los Angeles incluant South Central, Downtown, East Los Angeles, Pacoima, Compton, Vernon, South Gate et Huntington Park.

7 Mais, au total, Vergara ne cherche pas seulement une approche scientifique pour ellemême, son œuvre relève d'un engagement social et politique qui affirme clairement un point de vue engagé. Cet engagement se fait selon des modalités esthétiques (l'archive numérique) dont il montre la cohérence à la fois scientifique et politique, si on veut bien comprendre que ce dernier mot relève chez lui d'un désir de vivre ensemble selon des règles communes à tous.

8 Une question reste pendante : pourquoi un jeune émigré chilien se lance-t-il dans un tel projet de travail, pour toute une vie? Il semble que la réponse tient dans sa biographie, que Vergara ne manque pas de rappeler en préalable à l'ouvrage ci-dessus cité. C'est par là qu'il faut donc débuter.

\section{A. De la pauvreté au ghetto}

9 Camilo José Vergara est né à Santiago du Chili, en 1944. À Rengo, une petite ville du centre du Chili, son père achète en 1951 une maison en adobe et plante des arbres fruitiers. Ses grands-parents sont des fermiers aisés qui vendent leur production fruitière en Europe et aux États-Unis depuis les années 1930. Il est élevé par une gouvernante française et a une vie facile et confortable. Mais au cours de son adolescence, il voit tous les objets précieux de la maison, comme les couverts en argent, disparaître les uns après les autres, vendus à des connaissances ou au prêteur sur gages. Alors que son père sombre dans l'alcoolisme, les membres de sa famille et les amis viennent en aide à la famille, payant l'école des enfants et donnant vêtements et magazines usagés : "Adolescent, je vivais dans la peur de l'abjection de la pauvreté alors que les circonstances semblaient s'aggraver sans cesse ${ }^{1}$.» (Vergara C.J., $1995: \mathrm{x}$ ) Lorsqu'il revient sur les lieux trente ans 
plus tard, la maison a disparu et Vergara explique : «Maintenant je comprends mieux les sentiments ressentis par les banlieusards qui se rendent dans leur ancien quartier, pour découvrir qu'il est devenu méconnaissable."

En 1965, à l'âge de vingt-et-un ans, il entre à l'université. Il achète un appareil photo et photographie la vie de son campus de l'Université Notre-Dame, à South Bend, Indiana. Il photographie aussi la ville, surtout le quartier où il réside, un ghetto où vivent les Noirs et quelques centaines de familles mexicaines.

11 Après l'université, il rejoint New York, où il fait de la photographie de rue pendant trois ans, principalement dans le Lower East Side, le Bronx et Brooklyn. Il y photographie les enfants qui jouent, les chiens qui copulent, les petites filles noires qui jouent à la poupée, les écoliers latino-américains qui font l'école buissonnière, et les juifs pauvres qui se tiennent devant les entrées d'immeubles. Alors qu'il est bien conscient de l'impasse dans laquelle le mettent ces photos qui repassent sur celles d'autres photographes, il décide de reprendre des études de sociologie à l'Université Columbia, où il obtiendra en 1977 une maîtrise. C'est là qu'il prend de la distance, qu'il articule conception et pratique. Photographiant sans cesse, c'est le moment où il développe une méthode pour enregistrer les transformations urbaines considérables qui se déroulent sous ses yeux. Tout cela l'entraîne en priorité, dès 1977, vers les appartements du ghetto auquel il semblait destiné et dont les pièces sombres et décrépies accueillent des familles entières. Ainsi regarde-t-il le ghetto comme une part de sa vie personnelle, ce qui n'est pas sans lui poser question: "Pourquoi une telle obsession à enregistrer ce qui décline, se désintègre et tombe en ruines?». Il est alors conscient de la nécessité d'enregistrer, non pas les événements, les crimes, les incendies, les manifestations de protestations qui font habituellement les titres des journaux et de la télévision, mais les blocks, les immeubles, les rues dans leurs transformations incessantes, afin d'en préserver la mémoire. Ainsi estce une autre idée de la mobilité des lieux que celle des événements qui l'intéresse, une autre actualité en mouvement sur le moyen et le long terme.

\section{B. Objets du travail}

Dans Dead Cities, Mike Davis note :

Le ghetto des années 1970 et 1980 était abandonné à la fois intellectuellement, financièrement et fiscalement. À une exception près : l'observation photographique et le documentaire (non subventionné) de Camilo Vergara. L'auteur et photographe chilien était le "Dernier Homme" de l'Amérique en déréliction [...]. Cela peut paraître étrange, mais sans les milliers de photographies d'immeubles individuels et de quartiers prises au fil du temps par Vergara, nous ne disposerions aujourd'hui quasiment d'aucune archive scientifique et historique attestant du processus d'évolution du paysage de ghetto. (Davis M., 2009 : 128)

En quoi cette archive photographique pouvait-elle se constituer avec une valeur scientifique et historique? Si Mike Davis ébauche une vague réponse à cette question, il est cependant plus préoccupé par son objet, le lien entre les destructions de guerre et les ruines du ghetto américain. Je me suis donc tourné directement vers Vergara pour comprendre. En effet, Vergara, non seulement a mis en place un dispositif photographique que l'on peut, on va le voir, qualifier de scientifique, mais de plus il allie à son geste photographique un travail d'écriture dense issu de sa formation sociologique. 


\section{1) Les catégories}

13 Vergara définit à plusieurs reprises les catégories et les objets sur lesquels il compte travailler. Ces catégories ont évolué depuis la première définition qu'il donnait de son travail en 1995 :

J'étudie de près : 1 ) les zones et bâtiments minés par la consommation de drogue et son commerce; 2) les quartiers choisis par les sans-abri pour y vivre; 3) la gentrification - principalement dans les secteurs choisis pour l'implantation de logements sociaux ; 4) ceux choisis pour l'implantation des NIMBY [not in my back yard], à la fois les institutions et services (particulièrement les lieux sélectionnés pour les foyers pour sans-abri, les prisons, les lieux de traitement des drogués) ; 5) "les marges»-quartiers éloignés, coins oubliés, souvent manquant de représentation politique, le long des lignes de chemin de fer, des voies rapides, des cimetières et des zones industrielles. (Vergara C.J., 1995 : xiv)

En 2005, Vergara redéfinit sept points :

- Fortification,

- Ruins,

- Empty lots,

- Social containers,

- A Visual Language of Art and Advertisements,

- Public Service Billboards,

- Bringing Suburbanization to inner cities. (Vergara C.J., 2005)

Enfin sur le site de la Rutgers University, il ajoute maintenant aux dix catégories de 2005 les thèmes suivants :

- Store Front Churches,

- Revitalization projects that failed,

- The People Repair and Maintenance Industry. (Vergara C.J., 2005)

\section{2) La constitution d'une archive}

L'archive est l'objet final du travail de Vergara, et il la constitue de manière patiente au fil des années. De ce point de vue, par sa ténacité et par sa rigueur, son travail fait écho à celui des Becher. Mais Vergara vient du monde de la photo de rue, et la rigueur qu'il emploie n'est pas destinée à la prise de vue elle-même qui serait formalisée une fois pour toutes selon des points de vue parfaitement identiques. Il est plus perméable aux accidents, aux micro-événements qui se produisent devant lui à chaque nouvelle photo d'un même lieu : celui-ci l'invite à chaque fois à reconsidérer le point de vue précédent, les variations de points de vue enregistrant ainsi de manière très empirique les modifications topologiques des lieux et la présence des passants.

Sur un plan matériel, l'archive se constitue sur des supports différents, tout d'abord des diapositives couleur argentiques $35 \mathrm{~mm}$, puis des tirages sur papier et enfin, depuis les années 2000, des fichiers numériques. Actuellement, quatre grandes archives de l'œuvre de Vergara existent :

- son propre site (http://www.camilojosevergara.com/), très documenté et intitulé Tracking Time, présente un ensemble très important de photographies réparties selon les lieux documentés : Harlem, Gary, Chicago, Détroit, Camden, Los Angeles, Newark, Brooklyn, South Bronx, etc. On peut suivre ainsi un certain nombre de reconductions photographiques. Depuis ce site, on accède aussi à son compte Instagram sous le titre No Dry Bones https://www.instagram.com/nodrybones/) ; 
- le Getty Research Institute de Los Angeles lui a acheté une collection importante (une centaine de photographies, complétées par une centaine d'autres données par le collectionneur et producteur de cinéma Bruce Berman²), que Mike Davis évoque ainsi : » Les études temporelles approfondies du New American Ghetto Archive de Vergara - dont l'original a été acquis par le Getty Research Institute de Los Angeles - exposent, pour ainsi dire, le déclin urbain dans sa forme "canonique". » (Davis M., 2009 : 128);

- Vergara lui-même a fait un important dépôt à la Bibliothèque du Congrès à Washington, «Plus de 5000 photographies [à ce jour, 8000 photographies $^{3}$ ] ont déjà été transférées à la Bibliothèque, et l'archive complète devrait offrir plus de 10000 images allant des années 1970 aux années 2010 .» À cela s'ajoutent 287 tirages photographiques en couleur et 1840 positifs en couleurs, alors que les 10000 photographies attendues par la Bibliothèque se présentent sous forme de photographies numériques ;

- la Rutgers University a organisé un site présentant de façon interactive le travail de Vergara, sous le titre très explicite: Invincible cities ( https:// invinciblecities.camden.rutgers.edu/intro.html) ${ }^{5}$. Ce titre se réfère à une inscription sur la mairie de Camden ${ }^{6}$ dans le New Jersey tirée de Leaves of Grass de Walt Whitman (1867), mais évoque aussi celui d'Italo Calvino, Invisible cities (1972; 1974 pour la traduction en anglais). On pourrait également penser au livre de Ralph Ellison, Invisible Man (1952), qui conduirait à développer un aspect du travail de Vergara se rapportant à la question de l'invisibilité : » Je cherche donc à attirer l'attention vers ceux qui, jamais encouragés, restent dans leur quartier. » (Vergara C.J., 2017) Vergara introduit ce site par un texte qui présente l'ensemble de son travail et de sa démarche, bien que ce texte soit une compilation d'autres textes déjà publiés, et l'on peut remarquer que le titre de l'archive a changé, passant de The New American Ghetto Archive à A Visual Encyclopedia of The American Ghetto. Le site référence principalement des vues de Harlem, Camden, New Jersey et Richmond, Californie. Depuis la page d'accueil, plusieurs fonctionnalités peuvent être remarquées: agrandissement d'une image, apparition d'un plan de situation, repères permettant de retrouver une adresse précise de prise de vue, etc.

Il faut ajouter depuis 2007 l'usage intensif de Google Street View, à la fois pour repérer des lieux intéressants ou leur transformation, et pour capter de nouvelles images sans se déplacer. Sur Internet, Vergara consulte aussi les sites des restaurants, des églises, des entreprises, et suit régulièrement les forums de discussion; tout cela complète ou remplace les informations glanées sur place lors de ses déplacements: „ Google Maps exerce maintenant une véritable influence sur le choix des lieux que je visite, les photographies que je prends et les conversations avec les gens rencontrés sur place. " ( Vergara C.J., $2016: 3$ )

\section{Dispositifs photographiques}

\section{1) La méthode de Vergara : la reconduction}

17 C'est dans The New American Ghetto (1995), que Vergara définit le plus clairement sa méthode de travail, en dix points qu'il me paraît ici important de présenter :

1) J'enregistre d'abord les changements évidents par l'observation précise des images.

2) Je compare les aspects de villes différentes.

3) Jusqu'en 1986, je prenais habituellement les photos depuis le niveau de la rue, mais avec les dealers de crack vendant à tous les coins de rue, cela devint 
dangereux... J'ai commencé à chercher un résident pour m'accompagner. Mon attention à la sécurité m'a ouvert de nouvelles possibilités de travail. Je me suis tourné vers les agences publiques de logement, les bailleurs sociaux, les hôpitaux, et les universités pour m'aider... J'ai commencé à prendre systématiquement des photos depuis des points de vue élevés.

4) Les quartiers sont représentés à la fois depuis le niveau de la rue et à vue d'oiseau.

5) La collection d'images est organisée en réseau.

6) J'ai utilisé essentiellement la même approche pour documenter des communautés pauvres ou minoritaires.

7) Je ne dispose que de cinq jours par an pour photographier un lieu.

8) Je rôde dans toute la ville à la recherche de formes locales qui définissent le nouveau ghetto: institutions charitables, NIMBY, ruines, expressions graphiques, fortifications, enclaves.

9) Mes catégories thématiques et mes arguments trouvent leur origine dans les images et les expériences faites sur place.

10) En écrivant le texte, j'étais guidé par les gens que je rencontrais au cours des prises de vues et par d'autres à qui les images elles-mêmes m'ont mené. Certains devinrent mes référents et je les appelais fréquemment pour obtenir un avis. Numéros de téléphone laissés sur les murs, noms sur les façades de magasins et sur les voitures, cartes professionnelles données par les chauffeurs de taxi, messages laissés sur des panneaux, morceaux de papiers collés sur les panneaux électoraux, tout cela me fournissait de nouvelles informations et une meilleure compréhension de l'environnement. (Vergara C. J., 1995 : xiii-xiv)

Tout au long des années, Vergara retourne sur les mêmes «sites" photographiques, reconduisant les mêmes prises de vue des mêmes lieux, sans toutefois s'astreindre à une similitude parfaite. Depuis le milieu des années 2000, le système de classement et d'indexation des images électroniques Lightroom lui permet de complexifier pour ses photographies son système de classement à multiples entrées. À l'étude empirique sur le terrain succède donc la mise en place d'un outil analytique sous forme d'une archive interactive de toutes ces photographies. On remarque là l'influence d'une école sociologique empiriste dont Loïc Wacquant se réclame lui-même : » L'ambition empirique immédiate et l'objectif analytique ultérieur [...] sont étroitement liés. (Wacquant L., $2007: 6)$

Vergara reconnaît pourtant la complexité de sa méthodologie : «C'est devenu de plus en plus complexe de construire des réseaux d'images photo par photo. La documentation existante requiert constamment un travail de terrain pour rester en prise avec l'évolution phénoménale de nos villes (Vergara C. J., 1995 : xv). Afin de surmonter cette complexité apparait le principe de la reconduction photographique comme méthode. On connaît bien ce procédé qu'on rencontre régulièrement, notamment avec les cartes postales, et qui consiste à retourner sur un lieu documenté par une vieille photo afin d'en refaire le point de vue à l'identique. La pulsion comparatiste domine largement ce type de travail dont on pourrait n'apercevoir aucun intérêt autre qu'anecdotique. Mais avec Vergara tout change, car cette méthode de travail lui permet d'interroger les conditions sociales, économiques et politiques des changements permanents de la typologie du ghetto. Nous y reviendrons plus loin.

\section{2) Transversalité}

20 Au bout d'un certain nombre d'années de travail, Vergara remarque des points communs, des objets et figures récurrents d'un lieu de travail à l'autre et va les photographier puis 
les rassembler dans des montages dont le support privilégié est le livre. C'est ce que nous pouvons observer dans l'ouvrage publié en Allemagne chez Kerber en 2014. Nous y voyons un très bel ensemble de murals photographiés en divers lieux, comme Los Angeles, Gary, Détroit, New York, Newark, Camden, entre 1993 et 2014. On remarque là la continuation de l'esprit des grands muralistes mexicains, comme Diego Rivera et José Clemente Orozco, importé durant la Dépression dans les grandes villes américaines. Ici, les thèmes sont tout aussi politiques que les murals de Derry ou Belfast du côté républicain. On comprend combien les habitants des ghettos continuent de revendiquer une appartenance à la grande nation américaine alors qu'ils ont souvent le sentiment d'en être rejetés. Les figures de Martin Luther King, Malcolm X et Barack Obama sont les plus présentes, ainsi que celle de Mohamed Ali, dont Joyce Carol Oates soulignait « qu'il a imposé sa marque comme présence politique radicale» (Oates J.C., 2015 [1987]: 183) ${ }^{7}$. Ainsi la transversalité engage une autre sorte de montage : tandis que la reconduction mettait à plat le terrain de travail, la transversalité en fait ensuite ressortir les traits saillants, dans l'ordre du politique et du social.

\section{3) Photographie documentaire}

21 C'est précisément autour de ces questions politiques et sociales que cherche à agir Vergara, mettant en œuvre une photographie documentaire critique, dans la veine de celle créée à l'Université de Californie à San Diego au début des années 1970 par le groupe formé par Allan Sekula, Martha Rosler, Fred Lonidier ou Phel Stenmetz. Pour ces artistes, majoritairement photographes, il s'agit de mener deux combats esthétiques : d'une part lutter contre l'essentialisation formaliste de la photographie documentaire imaginée par John Szarkowski au MoMA de New York, et d'autre part dépasser le constat social jugé finalement inopérant des photographies de la FSA ou de la Photo League.

Pour Sekula notamment, alors qu'il constate avec une sorte d'effarement que l'art de son époque, conceptualisme ou minimalisme, ne porte presque pas de traces de la guerre du Vietnam, il faut revenir à un lien entre l'art et le monde qui lui est extérieur, et établir les ponts entre vie privée et scène politique et idéologique. Cette nouvelle position cherche à renouveler le genre documentaire, à en élargir les formes, à lui redonner un potentiel critique percutant. Le montage doit rester l'outil esthétique principal de ce renouveau, usant de télescopages, d'effets contradictoires, de mises en relation d'éléments opposés, afin de créer un espace mental que le spectateur puisse investir de ses propres pensées et analyses.

Vergara opère consciemment dans cette optique, ce qu'il confirme lorsqu'il écrit :

Des photographies qui ne dépeignent que le moment, qui perdent le sens global et qui sont construites autour de lumières dramatisées et de compositions puissantes qui occultent les détails importants, modèlent plus qu'elles n'enregistrent la réalité. Les images actuelles, éblouissantes, nous laissent le plus souvent à la surface des choses et de ce fait ne peuvent pas nous aider dans notre prise de conscience, encore moins promouvoir un changement social. (Vergara C.J., 1995 : xv)

Le travail de Vergara est politique : il veut non seulement enregistrer un fait social mais aussi promouvoir le changement. À ses yeux, cependant, deux écueils sont à éviter. Le premier concerne le genre très américain de la street photography, qu'il juge trop codifié après l'avoir pratiqué et en avoir éprouvé les limites : «Le paysage urbain de la pauvreté est beaucoup plus varié et riche que ce que nous en montre la street photography qui est centrée sur les gens. » (Vergara C.J., 1995 : xv) Pour lui, Helen Levitt a déjà fait ce genre de 
travail à New York, point n'est besoin de le refaire. Et, précisément, le moment où il abandonne la street photography coincide avec son retour à l'Université pour poursuivre ses études de sociologie.

Le deuxième écueil est celui du photojournalisme, et là, Vergara est on ne peut plus clair et rejoint en cela la virulente critique faite de ce milieu professionnel par Sekula. Il explique: "Les photojournalistes ont tendance à produire un travail si convenu qu'il échoue à susciter l'intérêt. Les images produites pour illustrer un texte existant, pour servir les besoins d'un client, ou pour confirmer les conceptions d'un rédacteur en chef, manquent de l'envergure suffisante pour capter et expliquer la réalité urbaine.» (Vergara C.J., 1995: xv) Vergara, montrant que les photojournalistes produisent un travail clairement inféodé à leurs commanditaires, décrit ici les trois principaux cas de figure auxquels ceux-ci sont soumis alors qu'à la même époque le sociologue Howard Becker le souligne de manière très lapidaire : «Le photojournalisme est le produit des contraintes économiques qui pèsent sur le journalisme en général. » (Becker H., 2001 : 335)

26 S'opposant au photojournalisme par l'usage d'une photographie documentaire critique, Vergara opère selon une attitude heuristique, perméable par ses observations à ce qu'il enregistre sur le terrain. Tout ce qu'il y trouve non seulement lui donne des informations, mais lui donne aussi à réfléchir. Tous ces éléments sont les pièces d'un gigantesque montage urbain à partir de quoi construire sa propre pensée. C'est ce lieu, le ghetto, ce lieu où naît la pensée de Vergara, qu'il faut donc interroger comme le contexte essentiel de son travail, dans toute sa profondeur historique, afin de comprendre les enjeux véritables de son œuvre.

\section{Le contexte du ghetto}

\section{1) Évolution historique}

27 Le ghetto américain se constitue dans les grandes villes du nord du pays et de la côte Ouest à partir de la « grande migration » des Africains-Américains depuis les États du Sud, principalement des années 1910 aux années 1950, même si elle a continué ensuite, au moins jusqu'aux années 1970. C'est la poussée industrielle, avec la Première Guerre mondiale d'une part, et d'autre part les conditions désastreuses de vie dans les États du Sud, qui ont conduit à cet exode. Rapidement, des quartiers se peuplent d'AfricainsAméricains, ce qui fait fuir les populations blanches, alors qu'une autre émigration se produit après la Seconde Guerre mondiale, celle des Blancs des centres-villes vers les banlieues.

Pourquoi parle-t-on d'un ghetto plutôt que d'un « quartier défavorisé » comme nous les connaissons en France? Loïc Wacquant indique que le ghetto est une "formation sociospatiale compacte et clairement circonscrite dans laquelle les Noirs de toutes les classes étaient consignés et soudés entre eux par un large éventail d'institutions propres au groupe et à son espace réservé » (Wacquant L., 2007 : 7), sorte de société alternative imposée d'en haut par les pouvoirs publics et par toute une conjonction de facteurs sur lesquels je ne reviendrai pas ici. Comme Wacquant l'affirme: "La réalité du ghetto comme lieu physique, social et symbolique de la société étasunienne est largement façonnée - en fait imposée - de l'extérieur, du fait que ses habitants sont de plus en plus 
complètement dépossédés des moyens de produire leur propre identité individuelle et collective. » (Wacquant L., 2007 : 53)

Plusieurs moments de l'évolution du ghetto montrent les conditions de vie dégradées malgré la solidarité qui règne alors dans ces quartiers, où des institutions sociales alternatives sont encore vivaces. Les grandes émeutes de 1919 traversent beaucoup de grandes villes, comme Chicago. Dans cette ville, Carl Sandburg, dans un célèbre texte, donne un aperçu de la violence qui règne dans les quartiers noirs: «Huit bombes ou caisses de dynamite ont explosé au cours des cinq derniers mois sur le perron de bâtiments des quartiers sud de la ville, tous situés dans des rues adjacentes au secteur résidentiel communément appelé la "Black Belt", où la population est à peu près à $80 \%$ noire. » (Sandburg C., 2016 [1919] :107)

La grande crise des années 1930, est décrite par Caroline Rolland-Diamond dans cet aperçu tranchant :

La Federal Housing Administration mit en place [en 1934] une politique dite du redlining consistant à établir des cartes entourant de rouge les zones non éligibles pour les prêts garantis et à les transmettre aux banques, renforçant ainsi leurs réticences à accorder des crédits immobiliers à des familles noires. Au lieu d'être aidés comme les Blancs à devenir propriétaires, les Noirs américains durent rester locataires ou se contenter d'acheter des biens dans des quartiers peu prisés, les ghettos noirs, dont la densité continua de croître pendant la Dépression sous l'effet de l'exode rural des Noirs du Sud. (Rolland-Diamond C., 2016 : 138)

C'est ce que confirme la sociologue Henrika Kuklick sur le New York des années 1930 à propos de l'action négative de cette même FHA à Brooklyn: «La présence d'une seule famille noire dans un îlot d'immeubles suffisait, étant donné les objectifs du FHA, à noter l'îlot comme "noir", comme on peut le constater sur une carte de Brooklyn datant de mars 1939. » (Kuklick H, 2009 : 352)

\section{Vladimir Pozner écrit en 1938 :}

Harlem est la partie la plus congestionnée de la ville, celle où il y a le plus de taudis, où les loyers sont les plus élevés : généralement $20 \%$ au-dessus des prix courants. Les propriétaires savent bien que les Noirs ne peuvent pas aller loger autre part. Il est rare qu'une famille noire ne soit obligée de sous-louer une ou plusieurs pièces ; souvent, cinq à six personnes couchent dans la même chambre, et néanmoins le loyer représente un tiers, sinon la moitié du salaire. (Pozner V., 2009 [1938] : 37)

Dans les années 1960-1970, comme contrecoup de la lutte pour les droits civiques, la situation des ghettos se dégrade à mesure que les emplois disparaissent. C'est le déclin de grandes villes autrefois riches, dont l'emblème reste Détroit. Les «ouvriers noirs révolutionnaires " de la ville constatent d'eux-mêmes cet effondrement, comme le souligne Dan Georgakas à propos de la construction entre 1971 et 1977 du Renaissance Center :

La population noire portait également une grande partie du fardeau de la diminution des services publics, en particulier des transports en commun, quasi inexistants, et du système scolaire, au bord de la faillite. Des milliers de maisons dans la ville proprement dite furent abandonnées en raison de la corruption dans les institutions de location publiques et privées, faisant des tours de Détroit, qui avaient coûté plusieurs millions de dollars, une immense mascarade. (Georgakas D. et Surkin M., $2015: 24-25)$.

Cette description d'un habitat ravagé par la faiblesse de l'aide sociale est complétée par un autre aperçu de Julius Horwitz à Harlem dès 1970 : « Notre chambre est sur le côté de l'immeuble et j'entends les sacs d'ordures qui passent devant notre fenêtre pour aller 
atterrir dans la cour. Le tas d'ordures continue de grandir. À présent, on dirait une montagne... Il tombe tant d'ordures chaque jour. La nuit, nous entendons les bouteilles qui se fracassent contre le mur. » (Horwitz J., 2015 [1970] : 56)

Dans les années 1970-1990, Studs Terkel se livre à une enquête de grande envergure et publie de nombreux témoignages oraux, dont celui-ci, de Jim Capraro en 1990 dans les rues de Marquette Park à Chicago. Il est alors directeur général de la Great Southwest Development Corporation, une association de quartier du Southwest Side à Chicago: "Quand j'ai commencé à travailler ici, en 1976, autour il n'y avait que des immeubles vides et incendiés. C'était juste un désert, un endroit menaçant. Vous auriez dû voir les briques et les gravats noircis, les fenêtres barricadées. Les gens avaient peur de s'aventurer par ici. » (Terkel S., $2010: 186)$.

\section{2) L'hyperghetto}

36 C'est dans cette période des années 1970-1990 que le ghetto se déstructure pour conduire à ce que Wacquant, repris par Vergara, nommera à la fin des années 1990 l'hyperghetto. Arrivé à la fin des années 1980 à Chicago pour faire sa thèse, Wacquant s'est installé à la frange du West Side. Il décrit l'hyperghetto en ces termes :

L'hyperghetto de la fin du siècle, nouvelle configuration spatiale et organisationnelle décentrée, caractérisée par la double ségrégation de race et de classe dans le contexte du double retrait de la Ceinture noire historique du marché du travail et de l'État-providence, retrait qui nécessite et suscite en retour le déploiement d'un appareil policier et pénal intrusif et omniprésent. (Wacquant L., $2007: 7$ )

En effet, dès le début des années 1980, toutes les institutions qui pouvaient maintenir une solidarité, une cohérence interne au ghetto et le rendre sinon acceptable, du moins " vivable » pour ses habitants, ont disparu ou sont devenues complètement inopérantes, que ce soit l'école, les transports ou l'hôpital, pour ne citer que les plus cruciales. Wacquant donne alors une description des lieux totalement désespérante : «Bâtiments abandonnés, terrains vagues jonchés de débris et d'ordures, trottoirs défoncés, devantures d'officines religieuses condamnées, et décombres carbonisés de magasins s'alignent sur des kilomètres et des kilomètres dans ces zones en déshérence..." (Wacquant L., 2007 : 58-59). À propos des années 1980, Caroline Rolland-Diamond désigne la source de cette déréliction :

Le gouvernement durcit aussi considérablement les critères d'éligibilité aux allocations familiales et réduisit le budget fédéral alloué au logement social, entraînant la destruction annuelle d'un millier de logements et l'interdiction de l'usage de 70000 autres. Enfin, l'administration Reagan supprima les programmes d'aide technique et financière directes aux quartiers urbains défavorisés. La mise en œuvre de cette politique se traduisit par une détérioration catastrophique des conditions de vie des habitants des ghettos. (Rolland-Diamond C., $2016: 439$ )

C'est dans ce contexte que le projet de Camilo Vergara voit le jour. Mais il faut amender ces descriptions et analyses déprimantes pour comprendre le sens réel de son travail tel qu'il débuta en 1977. De manière très critique envers l'École de Chicago, Henrika Kuklick donne une vision plus complète de la situation de l'hyperghetto lorsqu'elle indique comment celui-ci est soumis au flux et reflux immobilier :

Car la ville est perçue comme une frontière en perpétuel mouvement, constamment soumise à des cycles de dévastation et de construction. Par conséquent, alors même qu'ils apparaissent comme les instruments de forces historiques inexorables, les urbanistes ont constamment l'occasion d'élever des monuments tangibles à leur 
pouvoir personnel. Et la sociologie évolutionniste a consacré une stratégie professionnelle viable pour le bureaucrate en tant que tel en justifiant les relations privilégiées des fonctionnaires avec des clients puissants, en leur permettant de négocier la perpétuation de leur survie professionnelle. » (Kuklick H., 2009 : 366)

C'est de ce mouvement perpétuel, au gré des intérêts financiers, dont il retrouvait l'équivalent dans le photojournalisme qu'il dénonçait, que l'œuvre de Vergara rend compte: il s'agit non pas d'une catastrophe généralisée et permanente, mais d'une succession de vagues qui alternent construction et destruction, aussi bien immobilières que culturelles (Vergara C.J., 2016). En effet, Vergara travaille sur la culture visuelle de l'hyperghetto pour montrer la richesse culturelle et identitaire actuelle de ses habitants, ce qui notamment infirme la position de Loïc Wacquant ${ }^{8}$.

C'est en cela que la méthode qu'il met en place, croisant reconduction et transversalité dans une perspective documentaire critique, prend tout son sens sociologique, économique et politique. Il ne s'agit pas de faire des relevés topographiques de lieux considérés comme des paysages en mouvement, mais d'être au plus près, sur la longue durée, de ces vagues successives qui reflètent les différentes politiques et opérations de prédation et de corruption appliquées à l'inner city. Il entend enfin montrer que l'hyperghetto n'est pas forcément un lieu de désespérance absolue alors qu'il enregistre de nombreuses manifestations esthétiques dans les rues, manifestations dont il montre la variété et la richesse. C'est notamment ce qui s'affirme dans son dernier livre Detroit Is No Dry Bones quand il dit: « Detroit Is No Dry Bones montre comment l'on peut s'adapter dans sa lutte pour survivre dans les quartiers isolés de la ville, mais aussi développer une véritable culture visuelle - questions jugées trop peu importantes pour recevoir l'attention du public.» (Vergara C.J., 2017)

\section{Conclusion : les machines de guerre urbaines}

Deux mots-clés restent indispensables pour Vergara: adaptation et survie ${ }^{9}$. Deux mots dont il faudrait développer les significations, et dont la résultante pourrait se trouver dans ce que Deleuze a appelé «machines de guerre » et que Manola Antonioli commente ainsi :

Les «machines de guerre » sont à mes yeux tous les dispositifs d'ordre politique, social, artistique, philosophique, etc. qui, pour reprendre une expression de l'anthropologue britannique Tim Ingold, échappent à l'autorité omniprésente de la ligne droite ou de la "rectilinéarité », [...] elles caractérisent tous les savoirs, toutes les techniques et les pratiques que Deleuze et Guattari appellent « mineures » par oppositions aux sciences, aux techniques et aux formes de pouvoir « majeures » et institutionnalisées. (Antonioli M., 2015)

Considérant son intérêt pour tous les artefacts produits localement par les habitants, nous pouvons alors voir le travail de Vergara comme une machine de guerre urbaine, même si les institutions ont tendance à euphémiser et à ramener à leurs catégories ce travail exemplaire : ainsi, dans la production de Vergara, ces institutions ont-elles acheté en priorité les photographies de cimetières et celles des Twin Towers avant leur destruction, montrant combien elles restent ancrées dans une idée de morbidité, et de l'archive comme patrimoine.

Si l'on suit le commentateur de Deleuze, Vincent Jacques, «le concept de machines de guerre caractérise des "agencements collectifs nomades". Ce concept concerne ainsi "des mouvements politiques à l'intérieur même de l'État, des œuvres qui bouleversent règles 
et académisme, la pensée qui combat les formes de l'institution en elle..." » (Jacques V., 2014). Le nomadisme du photographe parait évident, et c'est bien en investissant de ses photographies des institutions prestigieuses qu'il fait valoir sa pensée critique. En cela, Rutgers University, en construisant ce site web, permet au visiteur de déployer son propre regard critique, de remodeler s'il le souhaite les catégories de Vergara et de donner directement son avis.

Le travail de Vergara consiste donc en une démarche d'entrisme à l'intérieur des institutions étatiques, démarche qui n'est pas seulement une observation participative des ghettos, mais un moyen de faire sortir celui-ci du carcan dans lequel l'ultralibéralisme violent l'a enfermé depuis des décennies. Comme le souligne Vincent Michel, «la spatialisation du capitalisme dans sa domination contribue elle-même à recréer en permanence des "zones périphériques" " (Michel V., 2015: 9) et c'est cela que met en évidence le travail de Vergara : la mouvance permanente de ces zones périphériques, leur agitation qui résiste envers et contre tout à l'écrasement définitif, ce pourquoi Vergara a appelé ces zones les cités invincibles. Ce travail n'est donc pas négatif, tourné comme tant d'autres vers le catastrophisme, par exemple chez des photographes comme Robert Polidori, Thomas Jorion ou encore Marchand et Meffré. Il propose au contraire une orientation et des moyens de lutte. Michel ajoute, et l'on est tenté de le suivre: "Le processus d'intervention des "machines de guerre" est vu non comme le recours à une arme belligérante, mais comme un système alternatif à la pensée dominante, élaboré par des groupes sociaux inédits et séparés des procédures étatiques officielles, et donc tout à fait déterminant pour ceux qui veulent concevoir et réaliser des transformations. » (Michel V., 2015: 9) On le sait, les Africains-Américains, groupe social rejeté des procédures officielles, ont une patience à nulle autre pareille... alors que tout est fait depuis l'extérieur pour détruire systématiquement toute velléité de construction alternative. Ce qui se profile pour eux sous la présidence de Trump, avec le souvenir de l'ère Reagan, est pour le moins inquiétant. On peut craindre une reconduction des années 1980, sans doute encore plus décomplexée.

\section{BIBLIOGRAPHIE}

Antonioli, Manola (dir.), Machines de guerre urbaines, Paris, Loco, 2015.

Becker, Howard, « Sociologie visuelle, photographie documentaire et photojournalisme : tout (ou presque) est affaire de contexte », Communications, vol. 71, n 1, 2001, p. 333-351.

Benjamin, Walter, «Paralipomènes et variantes des Thèses sur le concept d'histoire », Écrits français, Paris, Gallimard, 1991 [1940], p. 356.

Davis, Mike, Dead Cities, Paris, Les Prairies ordinaires, 2009 [2003].

Georgakas, Dan et Surkin, Marvin, Detroit : pas d'accord pour crever, Marseille, Agone, 2015 [1975].

Horwitz, Julius, Journal d'une fille de Harlem, Paris, Le Seuil, coll. « Points », 2015 [1970].

Jacques, Vincent, Deleuze pas à pas, Paris, Ellipses, 2014.

IdeAs, 13 | 2019 
Kuklick, Henrika, « L'École de Chicago et la politique de planification urbaine ", in L'École de Chicago. Naissance de l'écologie urbaine, textes traduits et présentés par Isaac Joseph et Yves Grafmeyer, Paris, Flammarion, coll. « Champs essais », 2009.

Michel, Vincent, « Fabriquer la paix civile », in Antonioli, Manola (dir.), Machines de guerre urbaines, op. cit.

Oates, Joyce Carol, De la boxe, Auch, Tristram, 2015 [1987].

Pozner, Vladimir, Les États-Désunis, Montréal, Lux, 2009 [1938].

Rolland-Diamond, Caroline, Black America, Paris, La Découverte, 2016.

Sandburg, Carl, Les Émeutes raciales de Chicago. Juillet 1919, Paris, Anamosa, 2016 [1919].

Terkel, Studs, Race, histoires orales d'une obsession américaine, Paris, Éditions Amsterdam, 2010.

Vergara, Camilo José, The New American Ghetto, New Brunswick, Rutgers University Press, 1995.

Vergara, Camilo José, Images as a Tool of Discovery, site de la Rutgers University, Camden, New Jersey (https://invinciblecities.camden.rutgers.edu/intro.html), 2005.

Vergara, Camilo José, Detroit Is No Dry Bones: The Eternal City of the Industrial Age, Ann Harbor, The University of Michigan Press, 2016.

Vergara, Camilo José, « Détroit n'est pas complètement desséché », in L'œil de la photographie, mis en ligne le 6 mars 2017 : http://www.loeildelaphotographie.com/fr/2017/03/06/

article/159940905/camilo-vergara-detroit-is-no-dry-bones/

Wacquant, Loïc, Parias urbains, Paris, La Découverte, 2007.

\section{Autres ouvrages de Camilo Vergara}

Vergara, Camilo José et Jackson, Kenneth, Silent Cities, Princeton, Princeton Architectural Press, 1989.

Vergara, Camilo José, El Nuevo Mundo, The Landscape of Latino Los Angeles, Los Angeles, Southern California Studies Center, 2000.

Vergara, Camilo José, Unexpected Chicagoland, textes de Camilo José Vergara et Timothy J. Samuelson, New York, The New Press, 2001.

Vergara, Camilo José, American Ruins, New York, The Monacelli Press, 2003.

Vergara, Camilo José, Subway Memories, New York, The Monacelli Press, 2004.

Vergara, Camilo José, How The Other Half Worships, New Brunswick, Rutgers University Press, 2005. Vergara, Camilo José, Twins Towers Remembered, Princeton, Princeton Architectural Press, 2005. Vergara, Camilo José, Harlem, The Unmaking of a Ghetto, postface de Timothy J. Gilfoyle, Chicago, The University of Chicago Press, 2013.

Vergara, Camilo José, Tracking Time - Documenting America's Post-Industrial Cities, Bielefield, Kerber, 2014.

\section{Sites}

http://www.camilojosevergara.com/ 
https://www.instagram.com/nodrybones/

http://loc.gov/rr/print/coll/camilo-vergara-photographs.html

https://invinciblecities.camden.rutgers.edu/intro.html

\section{NOTES}

1. Sauf indication contraire, toutes les traductions sont de l'auteur.

2. Correspondance de l'auteur avec Camilo Vergara, 2 mars 2017.

3. Ibid.

4. Site de la Bibliothèque du Congrès à Washington, http://loc.gov/rr/print/coll/camilo-vergaraphotographs.html.

5. Depuis cinq ans qu'il utilise Lightroom, Vergara ne collabore plus à ce site qui est donc resté au point mort. Correspondance de l'auteur avec Camilo Vergara, 2 mars 2017.

6. «I dream'd in a dream I saw a city invincible to the attacks of the whole of the rest of the earth; // I dream'd that was the new city of Friends; // Nothing was greater there than the quality of robust love, it led the rest; // It was seen every hour in the actions of the men of that city, // And in all their looks and words. » Walt Whitman, « I Dreamed in a Dream » (1867), Leaves of Grass. Philadelphia, David McKay, [1900], p. 141.

7. Sur son site, Vergara consacre un chapitre entier aux murals consacrés à Martin Luther King, sous le titre MLK Folk Murals, comprenant 81 photographies.

8. Correspondance avec Camilo Vergara le 2 mars 2017.

9. Correspondance avec Camilo Vergara le 22 février 2017. «Two key words for me [...]: Adaptations and Survival. »

\section{RÉSUMÉS}

Né au Chili en 1944, Camilo Vergara a suivi toute sa formation en sociologie aux États-Unis, à la suite de quoi il a décidé d'appliquer sa spécialisation en urbanisme à la photographie comme outil scientifique et politique d'investigation des ghettos noirs étasuniens. Depuis plus de quarante ans, il documente inlassablement les quartiers les plus délabrés des États-Unis, Harlem en premier lieu, mais aussi Détroit, Chicago, Camden et d'autres villes. Utilisant la forme de la reconduction photographique il quadrille les quartiers abandonnés en photographiant toujours les mêmes endroits, selon les mêmes procédures, année après année. Vergara est le seul photographe à avoir documenté scientifiquement la déréliction des quartiers pauvres des ÉtatsUnis. Le texte montre comment s'est déployé le travail de Camilo Vergara, quelles sont ses idées et concepts. Vergara évite tout misérabilisme ou tout sensationnalisme de la misère, pour déconstruire les situations politiques et sociales ayant mené à de tels états de déréliction dans le ghetto noir.

Born in Chile in 1944, Camilo Vergara studied sociology in the United States. He then applied his training to photography as a scientific and politic tool of investigation of black ghettos. For the past forty years he has documented America's most dilapidated neighborhoods, first of all Harlem, then Detroit, Chicago, Camden, and other cities. Year after year, he has used 
rephotography to document the abandoned districts taking pictures of the same places, using the same procedures. Vergara is the only photographer to have scientifically documented the dereliction of poor neighborhoods in the United States. This article shows how Camilo Vergara's work developed, and what his ideas and concepts are. Eschewing the presentation of poverty in any sensational way Vergara deconstructs the political and social situations that led to such states of dereliction in the black ghetto.

Nacido en Chile en 1944, Camilo Vergara se formó como sociólogo en Estados Unidos. Posteriormente, decidió aplicar su especialización de urbanismo a la fotografía, como herramienta científica y política de investigación de los guetos negros norteamericanos. Desde hace más de cuarenta años, trabaja documentando incesantemente los barrios más dañados de Norteamérica. En primer lugar Harlem, pero también Detroit, Chicago, Camden y otras ciudades. Utilizando la forma de la reconducción fotográfica, recorre los barrios abandonados, fotografiando siempre los mismos lugares, con los mismos procedimientos, año tras año. Vergara es el único fotógrafo que ha documentado científicamente la decadencia de los barrios pobres de los Estados Unidos. El texto muestra cómo se ha desplegado el trabajo de Camilo Vergara, un inmigrante chileno en Estados Unidos, cuáles son sus ideas y conceptos. Vergara evita todo miserabilismo y todo sensacionalismo de la miseria para desconstruir las situaciones políticas y sociales que dejaron en tal estado de abandono al gueto negro.

\section{INDEX}

Mots-clés : reconduction photographique, archive web, ghetto, exclusion, photographie documentaire, photographie de rue, ruine, discrimination

Palabras claves : reconducción fotográfica, archivo web, gueto, exclusión, fotografía documental, fotografía callejera, ruina, discriminación

Keywords : rephotography, web archive, ghetto, exclusion, documentary photography, street photography, ruin, discrimination

\section{AUTEUR}

\section{PHILIPPE BAZIN}

Né en 1954, Philippe Bazin a étudié la photographie à l'École nationale supérieure de la photographie d'Arles entre 1986 et 1989. Son travail artistique se développe sur les relations entre esthétique et politique. Parmi ses ouvrages récents : Les Coupes (2017), John Brown's Body (2016), Reconstruction (2014), Le Milieu de nulle part (2012), La Radicalisation du Monde (2009). Un livre sur la photographie, Pour une photographie documentaire critique, est paru en 2017. Il enseigne la photographie à l'École nationale supérieure d'art de Dijon et y coordonne le programme de recherche Travail, migrations et ruralité. 